\title{
Risk of Injury According to Attention Deficit Hyperactivity Disorder, Comorbid Mental Illness, and Medication Therapy
}

\author{
Authors \\ R. M. Merrill, S. M. Thygerson, C. A. Palmer \\ Affiliation \\ Brigham Young University, Provo, USA
}

Key words
ADHD
claims data
incidence
injury
retrospective cohort
risk

received $\quad 05.04 .2015$

revised $\quad 02.09 .2015$

accepted $\quad 05.10 .2015$

\section{Bibliography}

DOI http://dx.doi.org/ 10.1055/s-0035-1565132

Published online:

February 1, 2016

Pharmacopsychiatry 2016;

49: 45-50

(c) Georg Thieme Verlag KG

Stuttgart $\cdot$ New York

ISSN 0176-3679

\section{Correspondence}

\section{Ray M. Merrill}

Department of Health Science

Brigham Young University

2063 Life Sciences Building

Provo UT 84602

USA

Ray_Merrill@byu.edu

\section{Abstract}

$\nabla$

Introduction: We identify the risk of selected types of injuries among patients with ADHD or ADHD and comorbid mental illness. We also assess whether selected medications used by patients with ADHD increase the risk of comorbid mental illness or influence the association between ADHD and injury.

Methods: A retrospective cohort study design was conducted using medical claims data from the Deseret Mutual Benefit Administrators (DMBA). ADHD diagnosis, injury, medication, and demographic data were extracted from claims files during 2001-2013. Rate ratios were adjusted for age, sex, and calendar year.

Results: Patients with ADHD were 7.9 (95\% CI 7.6-8.2) times more likely to have psychosis, 5.5 (3.9-7.8) times more likely to have alcohol- or drug-induced psychosis, and 6.0 (5.9-6.2) times

\section{Introduction}

Attention deficit hyperactivity disorder (ADHD) is a behavioral problem in which individuals display symptoms of inattentiveness, hyperactivity, and impulsivity. There are multiple possible etiologies for the disorder, such as neuro-anatomic, neuro-chemical, central nervous system insults, environmental factors, and genetic origins $[1,2]$. Both children and adults with ADHD have a greater risk of injury [3-9]. For example, in a large insurance database study involving individuals aged 0 through 64 enrolled during 19982005, the rate of injuries was 1.6 times greater among those with ADHD compared with those without the disorder [6]. The increased rate of injuries among those with ADHD was greatest for those in the age group 0-4 years, followed by the age group 20-64 years. The study also showed that the severity and frequency of injuries was significantly positively associated with ADHD. more likely to have neurotic or personality disorder. Therapy with amphetamine was positively associated with neurotic or personality disorder (rate ratio $=1.08,1.02-1.15$ ); methylphenidate was negatively associated with neurotic or personality disorder $(0.90,0.84-0.97)$; and atomoxetine was positively associated with psychosis (1.33, 1.21-1.46), alcohol- or drug-induced psychosis (2.38, 1.04-5.43), and neurotic or personality disorder (2.38, 1.04-5.43). ADHD was associated with an increased risk of injury, with ADHD and comorbid mental illness having a stronger increased risk of injury. Psychostimulants ameliorated the increased risk of injury for patients with ADHD.

Conclusion: Patients with ADHD have an increased risk of injury, significantly more so for those with ADHD and comorbid mental illness. Psychostimulants can lower the risk of injury among patients with ADHD.

Attention deficit hyperactivity disorder is often accompanied by other mental health problems [10]. Mental illness associated with ADHD in adulthood includes psychosis, neurotic, and personality disorder [11]. In a study conducted in 2005, employed workers with ADHD had higher levels of anxiety, bipolar disorder, depression, alcohol or drug abuse, antisocial disorder, and oppositional defiant disorder [12]. This same pattern is also found in children with ADHD. National Center for Health Statistics data indicate that children and adolescents with ADHD have a 7.4 times greater risk of anxiety disorder, 8.0 times greater risk of depression, 8.7 times greater risk of an autism spectrum disorder, and 10.7 times greater risk for Tourette syndrome [13]. Treatment for ADHD may involve behavioral therapy, stimulant mediation (e.g., methylphenidate, dexmethylphenidate, and amphetamines), or non-stimulant medication (e.g., atomoxetine). Stimulant medication is the most common form 
of treatment. Stimulant compared with non-stimulant medication may affect the association between ADHD and injury differently. In general, the effect of ADHD medication on the association between ADHD and injury is not well understood. In this study we identify the risk of selected types of injury among patients with ADHD or ADHD and comorbid mental illness. We also assess whether selected medications used by patients with ADHD are associated with comorbid mental illness. Finally, we evaluate the association between ADHD and injury and between ADHD and comorbid mental illness and injury according to medication use.

\section{Methods}

$\nabla$

\section{Study population}

A retrospective cohort study was conducted with medical claims data from the Deseret Mutual Benefit Administrators (DMBA). DMBA is a health insurance company for employees of the Church of Jesus Christ of Latter-day Saints. The database includes information on employees, their spouses and dependent children. Most enrollees were dependent children (43.6\%), followed by contract holders (32.9\%) and spouses (23.6\%). Since 1970 , the DMBA has provided health insurance and retirement income to Church employees and their families. The current study was based on electronic claims data from 2001 through 2012, of which over $96 \%$ were members of the Latter-day Saint Church. In 2001 the database had 78220 enrollees. In 2013, this number increased to 91607 (reflecting an increase of $17.1 \%$ ).

There is little enrollee turnover from year to year $(\sim<5 \%$ per year). Most of the turnover occurs among young adults who lose eligibility for coverage under their parents and individuals who become eligible for Medicare at age 65. However many of those receiving Medicare continue to be enrolled in the DMBA. Approximately $46 \%$ of contract holders were employed by the Latter-day Saint Church education system; $21 \%$ of contract holders were maintenance and custodial workers; and the remaining $33 \%$ of contract holders worked in other capacities for the Church. Approximately $70 \%$ of enrollees resided in Utah, $11 \%$ in Idaho, $4 \%$ in other mountain states, $6 \%$ in Pacific states, $4 \%$ in central states, $3 \%$ in southern states, and $2 \%$ in northeastern states.

With regards to generalizing the results, there are no studies that have shown that Latter-day Saints, or religion in general, is protective against ADHD. There is also no evidence that Latterday Saints access health care differently than non-Latter-day Saints or are influenced differently by psychological disorders. However, Latter-day Saints have a health code that highly discourages alcohol drinking, tobacco smoking, or use of illegal drugs, which may lower the risk of certain injuries $[14,15]$.

The database was de-identified according to Health Insurance Portability and Accountability Act (HIPAA) guidelines and was exempt from the need for informed consent by the Institutional Review Board (IRB), which is a research ethics review committee. The study was approved by the IRB at the University of Utah.

\section{Data collection}

We examined the automated claims records from January 1, 2001 to December 31, 2013 and used the International Classification of Diseases, $9^{\text {th }}$ Revision, Clinical Modification (2006) codes to define injuries and ADHD [16]. DMBA has continued to use the $9^{\text {th }}$ revision in order to retain consistency in its codes, from the late 1970s to the current time. Incidence rates of ADHD were based on cases divided by the number of unique members enrolled in DMBA per year. We refer to this number of enrollees as person-years. A total of 1064768 person-years are represented in the study. A person was considered to be a case if in a given year they had a claim for ADHD from a psychologist, pediatrician, or psychiatrist and/or a prescription for methylphenidates [Ritalin, Ritalin SR, Ritalin LA, Concerta, Methylin, Methylin ER, Metadate ER, Daytrana, Quillivant XR Metadate CD], methamphetamine hydrochlorides [Desoxyn], dexmethylphenidates [Focalin], amphetamine and dextroamphetamine [brand name: Adderall, Adderall XR], dextroamphetamines [Dexedrine, Dextrostat], and lisdexamfetamines [Vyvanse], non-stimulant medications, such as atomoxetine [Strattera]. A person with multiple claims for ADHD in a given year was only counted once. Because of considerable overlap between sub-codes and lack of uniformity by providers in applying the sub-codes, all patients seen in a given year with an ICD-9 code of 314 regardless of sub-codes (e.g., 314.00, 314.01, and 314.20) were designated as having ADHD. Data on age, sex, year, injury claims, and pharmaceutical information were also used in this study.

Claims with diagnoses codes 800-959 were used to define injuries. Selected injury types and their corresponding codes are presented in $\bullet$ Table 1 . Minor injuries not requiring medical attention would not involve a claim and, therefore, are not reflected in the study. If an injury occurred in the same year as a diagnosis with ADHD, we assumed that ADHD influenced the incidence of that injury. Multiple injuries were possible and included in the analyses.

\section{ADHD medication}

Drugs used to treat ADHD (stimulants and non-stimulants) aim to improve attention, concentration, and productivity, and suppress impulsive behavior. Stimulant drugs are much more common than non-stimulants drugs for treating ADHD. These drugs, effective in about $70-80 \%$ of cases [17], include methylphenidates [Ritalin, Ritalin SR, Ritalin LA, Concerta, Methylin, Methylin ER, Metadate ER, Daytrana, Quillivant XR Metadate CD], methamphetamine hydrochlorides [Desoxyn], dexmethylphenidates [Focalin], amphetamine and dextroamphetamine [brand name: Adderall, Adderall XR], dextroamphetamines [Dexedrine, Dextrostat], and lisdexamfetamines [Vyvanse]. Non-stimulant medications, such as atomoxetine [Strattera], were first approved by the Food and Drug Administration in 2003, have fewer sideeffects than stimulants and work for up to $24 \mathrm{~h}$ [17], although they are not always as effective as stimulants.

\section{Mental health variables}

Two classifications of psychotic conditions were made. The first psychosis variable combined alcohol- (diagnosis codes 291) and drug- (292) induced psychosis. The second psychosis variable combined schizophrenic disorders (295), episodic mood disorders (296), paranoid states (297), other nonorganic psychoses (298), and psychoses with origin specific to childhood (299). A third mental health variable combined neurotic (300) and personality (301) disorders.

\section{Statistical techniques}

Counts, proportions, rates, and rate ratios were used to describe the results. Rates were calculated per 1000 person-years. Incidence rates and rate ratios were compared across the levels of age, sex, and calendar year using $95 \%$ confidence intervals. Non- 


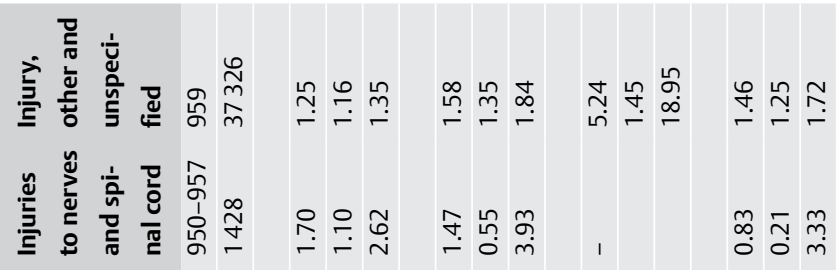

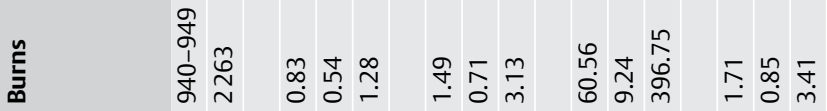

\section{迹㐫喜}

施 중

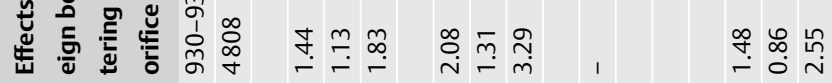

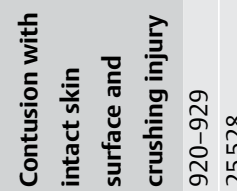

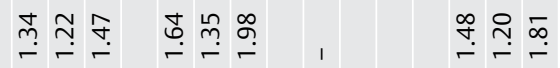

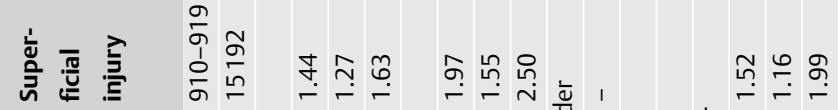

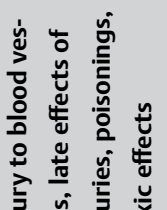

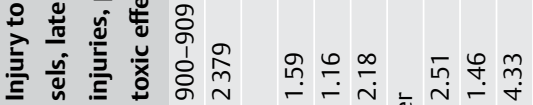

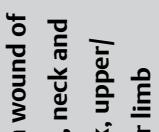

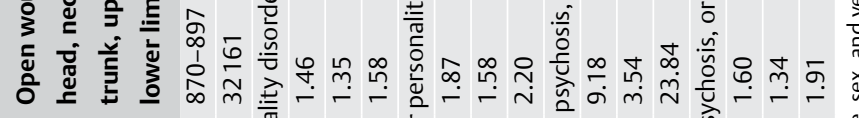

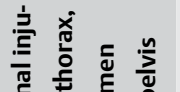

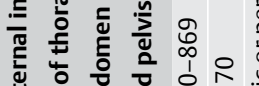

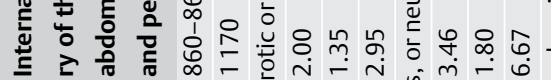

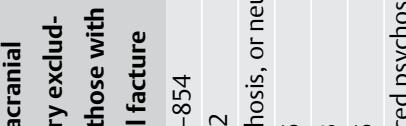

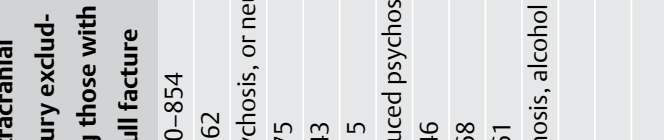

产

帝

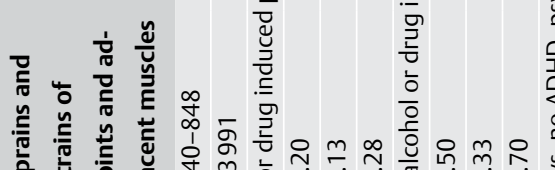

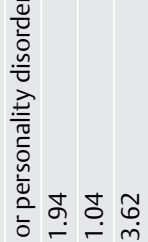

这

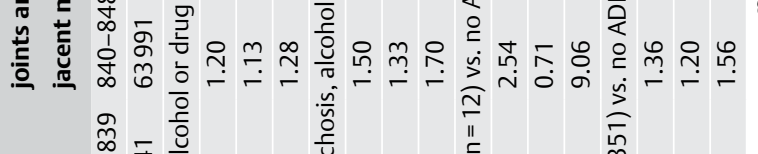

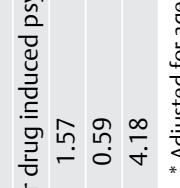

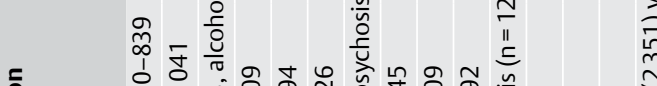

产

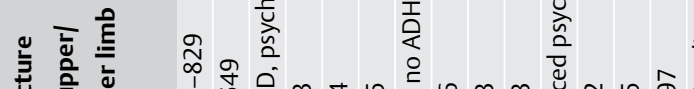

总

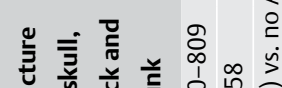

०

然

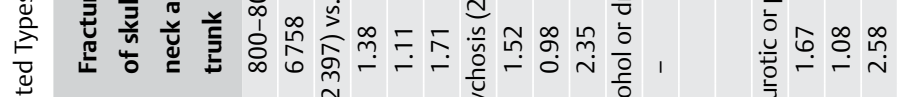

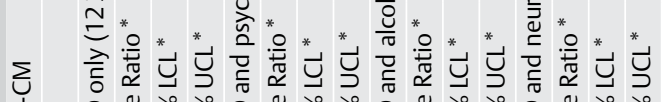


overlapping confidence intervals among rates indicated significant differences in the rates; estimated annual percent change in incidence rates indicated significance if the confidence interval did not overlap 0 ; and rate ratios indicated significant association between variables if the confidence interval did not overlap 1. Estimated annual percent change (EAPC) was estimated by regressing the natural log of the rate on calendar year. Then the estimated slope (b) was transformed as $100 \times\left(\mathrm{e}^{\mathrm{b}}-1\right)$. Two-sided tests of significance were used, based on the 0.05 level. Statistical analyses were derived from Statistical Analysis System (SAS) software, version 9.4 (SAS Institute Inc., Cary, NC, USA, 2012).

\section{Results}

$\nabla$

Mean age increased from $34.7(\mathrm{SD}=23.3)$ in 2001 to 36.2 $(S D=24.4)$ in 2013. The percentage of males and females remained constant ( $49 \%$ males and $51 \%$ females). The incidence rate of ADHD significantly increased from 2001 through 2013 (๑ Table 2), with an EAPC of 4.0 ( $p<0.0001)$. In 2013, the incidence rate of ADHD was greatest in the age range 10-17, followed by $18-24$, and then 25-34. The incidence rate of ADHD was almost twice as high for males as females. Overall there were 18618 patients with ADHD.

Among patients with ADHD, medication use varied across the study period ( $\bullet$ Fig. 1 ). For example, in 2001, methylphenidates were the most common medication, prescribed for $55.4 \%$ of patients, and amphetamine was prescribed $4.2 \%$ of the time. In contrast, in 2013 methylphenidates were prescribed $8.5 \%$ of the time compared with amphetamine prescribed $36.0 \%$ of the time. Use of amphetamine increased (EAPC $=10.0, p<0.0001$ ), methylphenidate use decreased (EAPC $=-12.1, \mathrm{p}<0.0001)$, other psychostimulants increased (EAPC $=9.9, \quad \mathrm{p}=0.0012)$, and atomoxetine decreased $(\mathrm{EAPC}=-13.7, \mathrm{p}<0.0001)$. Amphetamine use among patients with ADHD was greatest in the age groups $18-24,25-34$, and 35-44, whereas methylphenidates was greatest in the age groups $0-9,10-17$, and $45+$. There was little difference in medication use between males and females. In comparison to the 17.5 per 1000 patients with ADHD during 2001-2013, the rate (per 1000) of psychosis was 31.4, of alcohol- or drug-induced psychosis was 0.4 , and neurotic or personality disorder was 40.2 . Of those individuals with ADHD, 20.7\% had a medical claim for psychoses, $0.2 \%$ had a medical claim for alcohol- or drug-induced psychosis, and $21.0 \%$ had a medical claim for neurotic or personality disorder. Individuals with ADHD compared with those without the disorder were 7.9 (95\% CI 7.6-8.2) times more likely to have psychosis, 5.5 (3.9-7.8) times more likely to have alcohol- or drug-induced psychosis, and $6.0(5.9-6.2)$ times more likely to have neurotic or personality disorder, after adjusting for age, sex, and calendar year.

Among those with ADHD, use of amphetamine was not significantly associated with psychosis, or with alcohol- or druginduced psychosis. However, it was positively associated with an increased risk of neurotic or personality disorder (rate ratio adjusted for age, sex, and calendar year $=1.08,1.02-1.15$ ). Methylphenidates were also not significantly associated with psychosis or alcohol- or drug-induced psychosis, but were associated with decreased risk of neurotic or personality disorder $(0.90$, $0.84-0.97)$. Atomoxetine was significantly positively associated with psychosis $(1.33,1.21-1.46)$, alcohol- or drug-induced psychosis $(2.38,1.04-5.43)$, and neurotic or personality disorder $(2.38,1.04-5.43)$.

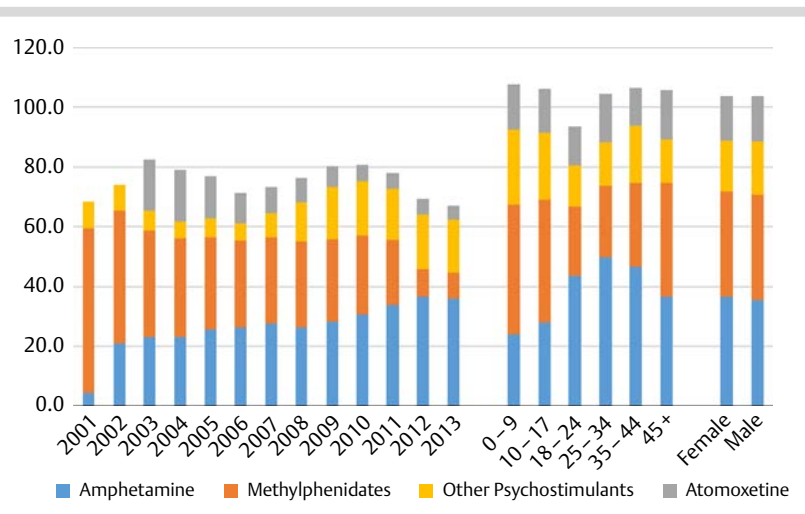

Fig. 1 Attention deficit hyperactivity disorder medication according to calendar year, age, and sex. (Color figure available online only).

Table 2 Incidence Rate of Attention Deficit Hyperactivity Disorder (ADHD) According to Age, Sex, and Calendar Year.

\begin{tabular}{|c|ccccc|}
\hline & Person- Years & No. & Rate/1 000 & $\mathbf{9 5} \% \mathbf{L C L}$ & $\mathbf{9 5 \%}$ UCL \\
\hline Year & & & & & \\
\hline 2001 & 78220 & 1012 & 12.9 & 1.2 & 1.4 \\
\hline 2002 & 78964 & 1103 & 14.0 & 1.3 & 1.5 \\
\hline 2003 & 79662 & 1236 & 15.5 & 1.5 & 1.6 \\
\hline 2004 & 80023 & 1291 & 16.1 & 1.5 & 1.7 \\
\hline 2005 & 78254 & 1308 & 16.7 & 1.6 & 1.8 \\
\hline 2006 & 77263 & 1243 & 16.1 & 1.5 & 1.7 \\
\hline 2007 & 80112 & 1299 & 16.2 & 1.5 & 1.7 \\
\hline 2008 & 81678 & 1362 & 16.7 & 1.6 & 1.8 \\
\hline 2009 & 82067 & 1482 & 18.1 & 1.7 & 1.9 \\
\hline 2010 & 82299 & 1539 & 18.7 & 1.8 & 2.0 \\
\hline 2011 & 86407 & 1766 & 20.4 & 1.9 & 2.1 \\
\hline 2012 & 88212 & 1912 & 21.7 & 2.1 & 2.3 \\
\hline 2013 & 91607 & 2065 & 22.5 & 2.2 & 2.4 \\
\hline Age (yrs), 2013 & & & & & \\
\hline $0-9$ & 13639 & 187 & 13.7 & 1.2 & 1.6 \\
\hline $10-17$ & 13082 & 619 & 47.3 & 4.4 & 5.1 \\
\hline $18-24$ & 12328 & 492 & 39.9 & 3.6 & 4.3 \\
\hline $25-34$ & 9164 & 238 & 26.0 & 2.3 & 2.9 \\
\hline $35-44$ & 8501 & 148 & 17.4 & 1.5 & 2.0 \\
\hline $45+$ & 34893 & 381 & 10.9 & 1.0 & 1.2 \\
\hline Sex, 2013 & & & & & \\
\hline Female & 46814 & 725 & 15.5 & 1.4 & 1.7 \\
\hline Male & 44793 & 1340 & 29.9 & 2.8 & 3.1 \\
\hline
\end{tabular}

LCL: Lower Confidence Limit; UCL: Upper Confidence Limit Source: Deseret Mutual Benefit Administration

An increased risk of injury for individuals with ADHD only compared with no psychosis, alcohol- or drug-induced psychosis, or neurotic or personality disorder is seen across the selected types of injury, with the exception of burns ( $\odot$ Table 1 ). ADHD in combination with psychosis compared with just ADHD has a greater risk for each type of injury, with the exception of injuries to nerves and spinal cord, where there is no significant difference in risk. Small numbers made it difficult to assess the association between the combination of ADHD and alcohol- or drug-induced psychosis and selected types of injury. However, there is some evidence of increased risk for selected types of injury. ADHD and neurotic or personality disorder compared with just ADHD has a greater risk for each type of injury, with the exception of internal injury of the thorax, abdomen, pelvis, and injuries to nerves and spinal cord, where there is no significant difference in risk. 
Table 3 Risk of Injury According to ADHD and Combinations of ADHD and Selected Mental Health Conditions.

\begin{tabular}{|c|c|c|c|}
\hline ADHD only & Rate Ratio & $95 \%$ LCL & $95 \%$ UCL \\
\hline Adjusted for age, sex, and year & 1.20 & 1.16 & 1.25 \\
\hline + Amphetamine (Adderall) & 1.10 & 1.06 & 1.14 \\
\hline + Methylphenidates (Ritalin) & 1.03 & 0.99 & 1.07 \\
\hline + Other psycho-stimulants & 1.00 & 0.96 & 1.04 \\
\hline + Atomoxetine (Strattera) & 0.99 & 0.95 & 1.03 \\
\hline \multicolumn{4}{|l|}{ ADHD and Psychosis } \\
\hline Adjusted for age, sex, and year & 1.48 & 1.38 & 1.59 \\
\hline + Amphetamine (Adderall) & 1.34 & 1.25 & 1.45 \\
\hline + Methylphenidates (Ritalin) & 1.25 & 1.16 & 1.35 \\
\hline + Other psycho-stimulants & 1.23 & 1.14 & 1.32 \\
\hline + Atomoxetine (Strattera) & 1.22 & 1.13 & 1.32 \\
\hline \multicolumn{4}{|c|}{ ADHD and Alcohol or Drug Induced Psychosis } \\
\hline Adjusted for age, sex, and year & 3.85 & 2.39 & 6.20 \\
\hline + Amphetamine (Adderall) & 3.38 & 2.12 & 5.38 \\
\hline + Methylphenidates (Ritalin) & 3.21 & 2.03 & 5.07 \\
\hline + Other psycho-stimulants & 3.16 & 1.99 & 5.02 \\
\hline + Atomoxetine (Strattera) & 3.58 & 2.12 & 6.06 \\
\hline \multicolumn{4}{|c|}{ ADHD and Neurotic or Personality Disorder } \\
\hline Adjusted for age, sex, and year & 1.38 & 1.28 & 1.49 \\
\hline + Amphetamine (Adderall) & 1.24 & 1.14 & 1.33 \\
\hline + Methylphenidates (Ritalin) & 1.17 & 1.08 & 1.26 \\
\hline + Other psycho-stimulants & 1.14 & 1.05 & 1.23 \\
\hline + Atomoxetine (Strattera) & 1.14 & 1.05 & 1.23 \\
\hline
\end{tabular}

ADHD only ( $n=12397)$, ADHD and psychosis $(n=2295)$, ADHD and alcohol- or drug-induced psychosis $(n=12)$, and ADHD and neurotic or personality disorder $(n=2351)$ are associated with an increased risk of injury in general, after adjusting for age, sex, and calendar year ( $\odot$ Table 3). ADHD only does not significantly increase the risk of injury after also adjusting for amphetamine and methylphenidates. The combinations of ADHD and psychosis, ADHD and alcohol- or drug-induced psychosis, and ADHD and neurotic or personality disorder each significantly increase the risk of injury, even after adjusting for age, sex, calendar year, and ADHD medication. The combination of ADHD and alcohol- or drug-induced psychosis has the strongest association with injury.

\section{Discussion}

$\nabla$

Studies have shown that both children and adults with ADHD have a greater risk of experiencing an injury $[6,18,19]$. The current study showed that the risk of injury is further increased when ADHD is accompanied by other comorbid mental illness. The use of psychostimulants ameliorated the association between ADHD and injury. Psychostimulants also contributed to lowering the association between ADHD and comorbid mental illness and injury, but they did not completely remove the increased risk of injury for these patients. The non-psychostimulant atomoxetine did not have a beneficial effect on lowering the association between ADHD and injury or ADHD and comorbid mental illness and injury.

The influence of medication therapy on ADHD or ADHD and comorbid mental illness and injury is complex. For example, ADHD combined with neurotic or personality disorder increased the risk of injury. Patients with ADHD and neurotic or personality disorder were less likely to experience an injury if they took amphetamine or methylphenidates. However, use of ampheta- mine or methylphenidates increased the risk of neurotic or personality disorder, which, in turn, was associated with an increased risk of injury. Nevertheless, the net effect of these medications in lowering the risk of injury among patients outweighs the increased risk they have on neurotic or personality disorder. In addition, atomoxetine was positively associated with psychosis, alcohol- or drug-induced psychosis, and neurotic or personality disorder, which were each associated with increased risk of injury. The net effect of this medication on the association between ADHD and alcohol- or drug-induced psychosis and injury was minimal.

This study assessed incidence rates and trends of ADHD rather than prevalence proportions. Studies assessing patterns in ADHD generally consider prevalence estimates and not incidence [20-22]. An advantage of using incidence rates (current ADHD cases) over prevalence proportions (previous and current ADHD cases) is that an association between incidence and injury is more direct. One older study that did use incidence rates of ADHD, based on DMBA data, covered the years 1998-2005 [6]. However, although that study did not find a significant increase in the incidence of ADHD for males or females, the current study, which goes 8 years beyond that study, did find a significant increase in ADHD, for both sexes, albeit more so for females.

Use of Adderall increased and use of Ritalin decreased over the study period. In 2006, 4.7 million persons aged 12 or older reported nonmedical use of Adderall, compared with 8.2 million in 2011 [23]. A concern is that some of this increase comes from greater use of Adderall for reasons other than medical need. One study found that $22 \%$ of adults who claimed to have ADHD exaggerated their symptoms [24]. They may have exaggerated their symptoms because they wanted a mental edge at work or school or because they really thought they had ADHD and wanted treatment. Unfortunately, nonmedical prescription stimulant users are more likely to take the medication in conjunction with alcohol, cigarettes, marijuana, ecstasy, cocaine, and participate in other high-risk behaviors [25]. The high level of comorbid mental illness observed in the current study is consistent with other studies $[12,13]$. Hence, there is a potential for drug interactions, which may help explain why medication did not significantly lower the risk of injury among patients with ADHD and comorbid mental illness. Although several studies have investigated the safety of ADHD stimulant drug use when combined with other medications and behaviors [26-31], more research is needed, especially in light of several case studies indicating an increased risk of psychosis and suicidal thoughts in users of Adderall and other stimulants [32-34].

The increased risk of selected types of injury among patients with ADHD is consistent with the previous DMBA study [6]. The current study further showed that individuals with ADHD and comorbid mental illness compared with ADHD only are at significantly greater risk of the selected injuries, with a few exceptions. This result is consistent with a recent large study based on the Dutch PHARMO record linkage system where use of ADHD and concomitant psychotropics significantly increased the risk of injuries compared with ADHD drug use only [35].

A limitation of this study is that claims associated with ADHD involved psychologists, pediatricians, and psychiatrists, not all of whom may have had the qualifications to diagnose ADHD or have conducted comprehensive cognitive assessment sufficient to rule out other diagnoses. There was sharp focus in major injuries that required a doctor's visit or hospitalization. The data did not include minor injuries. This information would have been 
hard to obtain and had the possibility of bias. In addition, although we were able to control for the potential confounding effects of age, sex, and year, we did not have data to allow us to control for socioeconomic status, race, alcohol drinking, smoking, or whether they used additional methods to cope with ADHD like behavior therapy. There was also a lack of information on the severity of the injuries and whether the rate ratios for ADHD differed by severity of injury.

\section{Conclusions}

$\nabla$

Attention deficit hyperactivity disorder is positively associated with an increased risk of several selected injuries. The greater risk of injury is significantly more pronounced for most of these injuries when comorbid mental health disorders also exist, especially the combination of ADHD and alcohol and drug induced psychosis. The positive association between ADHD only and injury was sufficiently controlled for by psychostimulants. The positive association between ADHD and comorbid mental illness and injury was improved, but not completely controlled for by psychostimulants. The non-psychostimulant atomoxetine did not show any benefit in reducing the risk of injury among ADHD patients.

\section{Conflict of Interest}

$\nabla$

The authors declare no conflict of interest.

\section{References}

1 Biederman J, Faraone SV. Attention-deficit hyper-activity disorder. Lancet 2006; 367: 210

2 Pearl PL, Weiss RE, Stein MA. Medical mimics: medical and neurological conditions simulating ADHD. Ann N Y Acad Sci 2001; 931: 97-112

3 Farmer JE, Peterson L. Injury risk factors in children with attention deficit hyperactivity disorder. Health Psychol 1995; 14: 325-332

4 Kessler RC, Lane M, Stang PE et al. The prevalence and workplace costs of adult attention deficit hyperactivity disorder in a large manufacturing firm. Psychol Med 2009; 39: 137-147

5 Leibson CL, Katusic SK, Barbaresi WJ et al. Use and costs of medical care for children and adolescents with and without attention-deficit/ hyperactivity disorder. JAMA 2001; 285: 60-66

6 Merrill RM, Lyon JL, Baker RK et al. Attention deficit hyperactivity disorder and increased risk of injury. Adv Med Sci 2009; 54: 20-26

7 Pastor PN, Reuben CA. Identified attention-deficit/hyperactivity disorder and medically attended, nonfatal injuries: US school-age children, 1997-2002. Ambul Pediatr 2006; 6: 38-44

8 Woodward LJ, Fergusson DM, Horwood LJ. Driving outcomes of young people with attentional difficulties in adolescence. J Am Acad Child Adolesc Psychiatry 2000; 39: 627-634

9 Xiang $H$, Stallones $L$, Chen $G$ et al. Nonfatal injuries among US children with disabling conditions. Am J Public Health 2005; 95: 1970-1975

10 Hodgkins $P$, Montejano L, Sasané R et al. Cost of illness and comorbidities in adults diagnosed with attention-deficit/hyperactivity disorder: a retrospective analysis. Prim Care Companion CNS Disord 2011; 13: doi:10.4088/PCC.10m01030

11 Biederman J. Impact of comorbidity in adults with attention-deficit/ hyperactivity disorder. J Clin Psychiatry 2004; 65 (Suppl 3): 3-7

12 Secnik K, Swensen A, Lage MJ. Comorbidities and costs of adult patients diagnosed with attention-deficit hyperactivity disorder. Pharmacoeconomics 2005; 23: 93-102
13 Larson K, Russ SA, Kahn RS et al. Patterns of comorbidity, functioning, and service use for US children with ADHD, 2007. Pediatrics 2011; 127: $462-470$

14 Hinckley GB. The scourge of illicit drugs. Ensign 1989; 48

15 The Church of Jesus Christ of Latter-day Saints. Published by The Church of Jesus Christ of Latter-day Saints, Salt Lake City, Utah, U.S.A. Section 1982; 89: 175-176

16 ICD-9-CM: International Classification of Diseases, $9^{\text {th }}$ Revision; Clinical Modification. $7^{\text {th }}$ ed. Los Angeles, California: PMIC; 2006

17 Center of Disease Control and Prevention, Division of Human Developmental, National Center on Birth Defects and Developmental Disabilities. Attention-deficit/hyperactivity disorder (ADHD). 2013; Retrieved from http://www.cdc.gov/ncbddd/adhd/treatment.html

18 Chou IC, Lin CC, Sung FC et al. Attention-deficit-hyperactivity disorder increases risk of bone fracture: a population-based cohort study. Dev Med Child Neurol 2014; 56: 1111-1116

19 Nigg JT. Attention-deficit/hyperactivity disorder and adverse health outcomes. Clin Psychol Rev 2013; 33: 215-228

20 Center of Disease Control and Prevention. Increasing prevalence of parent-reported attention-deficit/hyperactivity disorder among children - United States, 2003 and 2007. MMWR. 2010: 59: 1439-1443

21 Pastor PN, Reuben CA. Diagnosed attention deficit hyperactivity disorder and learning disability: United States, 2004-2006. Vital Health Stat 2008; 237: 1-14

22 Willcutt EG. The prevalence of DSM-IV attention-deficit/hyperactivity disorder: a meta-analytic review. Neurotherapeutics 2012; 9: 490-499

23 Center for Behavioral Health Statistics and Quality. 2012; [Tables 7.23A and 7.23B: in results from the 2011 national survey on drug use and health: detailed tables] National Survey on Drug Use and Health. Substance Abuse and Mental Health Services Administration. Retrieved from http://www.samhsa.gov/data/NSDUH/2011SummNatFindDetTables/ Index.aspx

24 Marshall $P$, Schroeder R, O'Brien $J$ et al. Effectiveness of symptom validity measures in identifying cognitive and behavioral symptom exaggeration in adult attention deficit hyperactivity disorder. Clin Neuropsychol 2010; 24: 1204-1237

25 McCabe SE, Knight JR, Teter CJ et al. Non-medical use of prescription stimulants among US college students: prevalence and correlates from a national survey. Addiction 2005; 100: 96-106

26 Kaskey GB. Possible interaction between an MAOI and "ecstasy". Am J Psychiatry 1992; 149: 411-412

27 Markowitz JS, Patrick KS. Pharmacokinetic and pharmacodynamic drug interactions in the treatment of attention-deficit hyperactivity disorder. Clin Pharmacokinet 2001; 40: 753-772

28 Markowitz JS, Morrison SD, DeVane CL. Drug interactions with psychostimulants. Int Clin Psychopharmacol 1999; 14: 1-18

29 Stotz G, Woggon B, Angst J. Psychostimulants in the therapy of treatment-resistant depression: review of the literature and findings from a retrospective study in 65 depressed patients. Dialogues Clin Neurosci 1999; 1 : 165-174

30 Utah Department of Health. Prescription opioid deaths in Utah. Violence and Injury Prevention Program 2012. 2011; Retrieved from http://useonlyasdirected.org/docs/RxOpioidDeaths.pdf

31 Van Waes $V$, Beverley J, Marinelli $M$ et al Selective serotonin reuptake inhibitor antidepressant potentiate methylphenidate (Ritalin)induced gene regulation in the adolescent striatum. Eur J Neurosci 2010; 32: 435-447

32 James SD. (2010, Nov8) Adderall abuse alters brain, claims a young life. ABC News Online. Retrieved from http://abcnews.go.com/Health/ MindMoodNews/adderall-psychosis-suicide-college-students-abusestudy-drug/story?id $=12066619$

33 Lakhan SE, Kirchgessner A. Prescription stimulants in individuals with and without attention deficit hyperactivity disorder: misuse, cognitive impact, and adverse effects. Brain Behav 2012; 2: 661-677

34 Surles LK, May HJ, Garry JP. Adderall-induced psychosis in an adolescent. J Am Board Fam Pract 2002; 15: 498-500

35 van den Ban E, Souverein P, Meijer W et al. Association between ADHD drug use and injuries among children and adolescents. Eur Child Adolesc Psychiatry 2014; 23: 95-102 